\title{
Sums of Involving the Harmonic Numbers and the Binomial Coefficients
}

\author{
Wuyungaowa, Sudan Wang
}

School of Mathematical Sciences, Inner Mongolia University, Hohhot, China

Email: wuyungw@163.com

Received 5 December 2014; accepted 22 May 2015; published 25 May 2015

Copyright (C) 2015 by authors and Scientific Research Publishing Inc.

This work is licensed under the Creative Commons Attribution International License (CC BY).

http://creativecommons.org/licenses/by/4.0/

(c) (i) Open Access

\section{Abstract}

Let the numbers $P(r, n, k)$ be defined by $P(r, n, k):=P_{r}\left(H_{n}^{(1)}-H_{k}^{(1)}, \cdots, H_{n}^{(r)}-H_{k}^{(r)}\right)$, where $P_{r}\left(x_{1}, \cdots, x_{r}\right)=(-1)^{r} Y_{r}\left(-0 ! x_{1},-1 ! x_{2}, \cdots,-(r-1) ! x_{r}\right)$ and $Y_{r}$ are the exponential complete Bell polynomials. In this paper, by means of the methods of Riordan arrays, we establish general identities involving the numbers $P(r, n, k)$, binomial coefficients and inverse of binomial coefficients. From these identities, we deduce some identities involving binomial coefficients, Harmonic numbers and the Euler sum identities. Furthermore, we obtain the asymptotic values of some summations associated with the numbers $P(r, n, k)$ by Darboux's method.

\section{Keywords}

Harmonic Numbers, Euler Sum, Riordan Arrays, Asymptotic Values

\section{Introduction and Preliminaries}

Let $Y_{r}$ be the exponential complete Bell polynomials and

In [1], Zave established the following series expansion:

$$
\begin{gathered}
P_{r}\left(x_{1}, \cdots, x_{r}\right)=(-1)^{r} Y_{r}\left(-0 ! x_{1},-1 ! x_{2}, \cdots,-(r-1) ! x_{r}\right) \\
\sum_{n=k}^{\infty}\left(\begin{array}{l}
n \\
k
\end{array}\right) P_{r}\left(H_{n}^{(1)}-H_{k}^{(1)}, \cdots, H_{n}^{(r)}-H_{k}^{(r)}\right) t^{n-k}=\frac{(-\ln (1-t))^{r}}{(1-t)^{k+1}}
\end{gathered}
$$


where $H_{n}^{(r)}=\sum_{k=1}^{n} \frac{1}{k^{r}}$ for $n, r=1,2, \cdots, H_{0}^{(r)}=0$ and $H_{n}^{(1)}=H_{n}$.

Spiess [2] introduced the numbers $P(r, n, k)=P_{r}\left(H_{n}^{(1)}-H_{k}^{(1)}, \cdots, H_{n}^{(r)}-H_{k}^{(r)}\right)$ and $P(1, n, 0)=H_{n}$, $P(r, n, k)=0$ for $n<r+k$; then Equation (1.1) is equivalent to

$$
\begin{gathered}
\sum_{n=k+r}^{\infty}\left(\begin{array}{l}
n \\
k
\end{array}\right) P(r, n, k) t^{n-k}=\frac{(-\ln (1-t))^{r}}{(1-t)^{k+1}} \\
\sum_{n=r}^{\infty}\left(\begin{array}{c}
n+k \\
k
\end{array}\right) P(r, n+k, k) t^{n}=\frac{(-\ln (1-t))^{r}}{(1-t)^{k+1}}
\end{gathered}
$$

where $P(0, n, k)=1, \quad P(1, n, k)=H_{n}-H_{k}, P(2, n, k)=\left(H_{n}-H_{k}\right)^{2}-\left(H_{n}^{(2)}-H_{k}^{(2)}\right)$,

$$
P(3, n, k)=\left(H_{n}-H_{k}\right)^{3}-3\left(H_{n}-H_{k}\right)\left(H_{n}^{(2)}-H_{k}^{(2)}\right)+2\left(H_{n}^{(2)}-H_{k}^{(2)}\right)
$$

The paper is organized as follows. In Section 2, we obtain some for $P(r, n, k)$ and binomial coefficients by means of the Riordan arrays. In Section 3, we establish some identities involving the numbers $P(r, n, k)$ and inverse of binomial coefficients. Finally, in Section 4, we give the asymptotic expansions of some summations involving the numbers $P(r, n, k)$ by Darboux's method. Due to [3] [4], a Riordan array is a pair $(d(t), h(t))$ of formal power series with $h_{0}=h(0)$. It defines an infinite lower triangular array $\left(d_{n, k}\right)_{n, k \in N}$ according to the rule

$$
d_{n, k}=\left[t^{n}\right] d(t)(h(t))^{k}
$$

Hence we write $\left\{d_{n, k}\right\}=(d(t), h(t))$. If $(d(t), h(t))$ is an Riordan array and $f(t)$ is the generating function of the sequence $\left\{f_{k}\right\}_{k \in N}$, i.e., $f(t)=\sum_{k=0}^{\infty} f_{k} t^{k}$. Then we have

$$
\sum_{k=0}^{\infty} d_{n, k} f_{k}=\left[t^{n}\right] d(t) f(h(t))=\left[t^{n}\right] d(t)[f(y) \mid y=h(t)]
$$

Based on the generating function (1), we obtain the next Riordan arrays, to which we pay particular attention in the present paper:

$$
\left\{\left(\begin{array}{l}
n \\
k
\end{array}\right) P(r, n, k)\right\}=\left(\frac{(-\ln (1-t))^{r}}{(1-t)}, \frac{t}{1-t}\right)
$$

Lemma 1 (see [5]) Let $\alpha$ be a real number and $L(z)=\ln \left(\frac{1}{1-z}\right)$. When $n \rightarrow \infty$,

$$
\begin{gathered}
{\left[z^{n}\right](1-z)^{\alpha} L^{k}(z) \sim \frac{1}{\Gamma(-\alpha)} n^{-\alpha-1} \ln ^{k} n, \quad(\alpha \notin\{0,1,2, \cdots\})} \\
{\left[z^{n}\right](1-z)^{m} L^{k}(z) \sim(-1)^{m} k m ! n^{-m-1} \ln ^{k-1} n, \quad\left(m \in \mathbb{Z}_{\geq 0}, k \in \mathbb{Z}_{\geq 1}\right)}
\end{gathered}
$$

\section{Identities Involving the Numbers $P(r, n, k)$ and Binomial Coefficients}

Theorem 1. Let $r, k \geq 0, n \geq 1$, then

$$
\sum_{j=r}^{n-1}\left(\begin{array}{c}
j+k \\
k
\end{array}\right) \frac{P(r, k+j, k)}{n-j}=\left(\begin{array}{c}
n+k \\
k
\end{array}\right) P(r+1, n+k, k)
$$


Proof. By (1), we have

$$
\begin{aligned}
\sum_{n=r}^{\infty}\left(\begin{array}{c}
k+n \\
k
\end{array}\right) P(r+1, n+k, k) t^{n} & =\frac{(-\ln (1-t))^{r+1}}{(1-t)^{k+1}} \\
& =\frac{(-\ln (1-t))^{r}}{(1-t)^{k+1}}(-\ln (1-t))=\sum_{n=r}^{\infty}\left(\begin{array}{c}
k+n \\
k
\end{array}\right) P(r, n+k, k) t^{n} \sum_{n=1}^{\infty} \frac{t^{n}}{n} .
\end{aligned}
$$

Comparing the coefficients of $t^{n}$ on both sides of (5), we completes the proof of Theorem 1 .

Recall that $P(0, n, k)=1$ Thus, setting $r=0,1,2$ in Theorem 1 gives the next three identities, respectively.

Corollary 1. Let $n \geq 1, \quad k \geq 0$, the following relations hold

$$
\begin{aligned}
& \sum_{j=0}^{n-1}\left(\begin{array}{c}
j+k \\
k
\end{array}\right) \frac{1}{n-j}=\left(\begin{array}{c}
n+k \\
k
\end{array}\right)\left(H_{n+k}-H_{k}\right), \\
& \sum_{j=0}^{n-1}\left(\begin{array}{c}
j+k \\
k
\end{array}\right) \frac{H_{j+k}}{n-j}=\left(\begin{array}{c}
n+k \\
k
\end{array}\right)\left(H_{n+k}^{2}-H_{n+k} H_{k}-H_{n+k}^{(2)}+H_{k}^{(2)}\right), \\
& \sum_{j=0}^{n-1}\left(\begin{array}{c}
j+k \\
k
\end{array}\right) \frac{H_{k+j}^{2}-H_{k+j}^{(2)}}{n-j}=\left(\begin{array}{c}
n+k \\
k
\end{array}\right)\left(\left(H_{n+k}-H_{k}\right)\left(H_{n+k}^{2}-H_{n+k}^{(2)}\right)-2 H_{n+k}\left(H_{n+k}^{(2)}-H_{k}^{(2)}\right)+2\left(H_{n+k}^{(2)}-H_{k}^{(2)}\right)\right) .
\end{aligned}
$$

Theorem 2. Let $r \geq 0, n \geq 1$, then

$$
\sum_{j_{1}=r}^{n-1} \sum_{j_{2}=r-1}^{j_{1}-1} \cdots \sum_{j_{r}=0}^{j_{r-1}-1}\left(\begin{array}{c}
j_{r}+k \\
k
\end{array}\right) \frac{1}{\left(n-j_{1}\right)\left(j_{1}-j_{2}\right) \cdots\left(j_{r-1}-j_{r}\right)}=\left(\begin{array}{c}
n+k \\
k
\end{array}\right) P(r+1, n+k, k)
$$

Proof. To obtain the result, make use of the Theorem 1.

Theorem 3. Let $n, r \geq 0, m \geq k$, then

$$
\sum_{k=0}^{n}\left(\begin{array}{l}
n \\
k
\end{array}\right)\left(\begin{array}{l}
m \\
k
\end{array}\right) P(r, n, k)=\left(\begin{array}{c}
n+m \\
m
\end{array}\right) P(r, n+m, m)
$$

Proof. Applying the summation property (2) to the Riordan arrays (3), we have

$$
\begin{aligned}
\sum_{k=0}^{n}\left(\begin{array}{l}
n \\
k
\end{array}\right)\left(\begin{array}{l}
m \\
k
\end{array}\right) P(r, n, k) & =\left[t^{n}\right] \frac{(-\ln (1-t))^{r}}{(1-t)}\left((1+y)^{m} \mid y=\frac{t}{1-t}\right) \\
& =\left[t^{n}\right] \frac{(-\ln (1-t))^{r}}{(1-t)^{m+1}}=\left(\begin{array}{c}
n+m \\
m
\end{array}\right) P(r, n+m, m),
\end{aligned}
$$

which is just the desired result.

Setting $m=n$ in Theorem 3 gives the next Corollary.

Corollary 2 Let $n, r \geq 0$, then

$$
\sum_{k=0}^{n}\left(\begin{array}{l}
n \\
k
\end{array}\right)^{2} P(r, n, k)=\left(\begin{array}{c}
2 n \\
n
\end{array}\right) P(r, 2 n, n)
$$

Corollary 3 Let $n \geq 0, m \geq k$, then

$$
\begin{aligned}
& \sum_{k=0}^{n}\left(\begin{array}{l}
n \\
k
\end{array}\right)\left(\begin{array}{l}
m \\
k
\end{array}\right)=\left(\begin{array}{c}
n+m \\
m
\end{array}\right), \\
& \sum_{k=0}^{n}\left(\begin{array}{l}
n \\
k
\end{array}\right)\left(\begin{array}{l}
m \\
k
\end{array}\right) H_{k}=\left(\begin{array}{c}
n+m \\
m
\end{array}\right) H_{n}-H_{n+m}+H_{m}, \\
& \sum_{k=0}^{n}\left(\begin{array}{l}
n \\
k
\end{array}\right)\left(\begin{array}{l}
m \\
k
\end{array}\right)\left(H_{k}^{2}+H_{k}^{(2)}\right)=\left(\begin{array}{c}
n+m \\
m
\end{array}\right)\left(\left(H_{n}-H_{n+m}+H_{m}\right)^{2}+H_{n}^{(2)}-H_{n+m}^{(2)}+H_{m}^{(2)}\right),
\end{aligned}
$$




$$
\begin{aligned}
\sum_{k=0}^{n}\left(\begin{array}{l}
n \\
k
\end{array}\right)\left(\begin{array}{l}
m \\
k
\end{array}\right)\left(H_{k}^{3}+3 H_{k} H_{k}^{(2)}+2 H_{k}^{(2)}\right)= & \left(\begin{array}{c}
n+m \\
m
\end{array}\right)\left(\left(H_{n}-H_{n+m}+H_{m}\right)^{3}+3\left(H_{n}-H_{n+m}\right)\left(H_{m}^{(2)}-H_{n+m}^{(2)}++H_{m}^{(2)}\right)\right. \\
& \left.+2\left(H_{n}^{(2)}-H_{n+m}^{(2)}+H_{m}^{(2)}\right)+3 H_{m}\left(H_{n}^{(2)}-H_{m+n}^{(2)}\right)\right) .
\end{aligned}
$$

Proof. Setting $r=0,1,2,3$ in Theorem 3 gives Corollary 3 .

Corollary 4. Let $n \geq 0$, then

$$
\begin{aligned}
& \sum_{k=0}^{n}\left(\begin{array}{l}
n \\
k
\end{array}\right)^{2}=\left(\begin{array}{c}
2 n \\
n
\end{array}\right) \\
& \sum_{k=0}^{n}\left(\begin{array}{l}
n \\
k
\end{array}\right)^{2} H_{k}=\left(\begin{array}{c}
2 n \\
n
\end{array}\right)\left(2 H_{n}-H_{2 n}\right) \text {, } \\
& \sum_{k=0}^{n}\left(\begin{array}{l}
n \\
k
\end{array}\right)^{2}\left(H_{k}^{2}+H_{k}^{(2)}\right)=\left(\begin{array}{c}
2 n \\
n
\end{array}\right)\left(\left(2 H_{n}-H_{2 n}\right)^{2}+2 H_{n}^{(2)}-H_{2 n}^{(2)}\right), \\
& \sum_{k=0}^{n}\left(\begin{array}{l}
n \\
k
\end{array}\right)^{2}\left(H_{k}^{3}+3 H_{k} H_{k}^{(2)}+2 H_{k}^{(2)}\right)=\left(\begin{array}{c}
2 n \\
n
\end{array}\right)\left(\left(2 H_{n}-H_{2 n}\right)^{3}+2\left(2 H_{n}^{(2)}-H_{2 n}^{(2)}\right)\right. \\
& \left.+3\left(H_{n}-H_{2 n}\right)\left(2 H_{n}^{(2)}-H_{2 n}^{(2)}\right)+3 H_{n}\left(H_{n}^{(2)}-H_{2 n}^{(2)}\right)\right) .
\end{aligned}
$$

Proof. Setting $r=0,1,2,3$ in Corollary 2 yields Corollary 4 .

Theorem 4. Let $n \geq r, \quad m \geq 2$, then

$$
\sum_{k=0}^{n}\left(\begin{array}{l}
n \\
k
\end{array}\right)\left(\begin{array}{l}
m \\
k
\end{array}\right) k P(r, n, k)=m\left(\begin{array}{c}
n+m-1 \\
n+1
\end{array}\right) P(r, n+m-1, m-2)
$$

Proof. which is just the desired result.

Setting $m=n$ in Theorem 4 gives the next Corollary.

Corollary 5. Let $n, r \geq 0$, then

$$
\sum_{k=0}^{n}\left(\begin{array}{l}
n \\
k
\end{array}\right)^{2} k P(r, n, k)=n\left(\begin{array}{c}
2 n-1 \\
n+1
\end{array}\right) P(r, 2 n-1, n-2)
$$

Corollary 6. The substitutions $r=0,1,2,3$ in Theorem 4 gives the next four identities, respectively.

$$
\begin{aligned}
& \sum_{k=0}^{n}\left(\begin{array}{l}
n \\
k
\end{array}\right)\left(\begin{array}{l}
m \\
k
\end{array}\right) k=m\left(\begin{array}{c}
n+m-1 \\
n+1
\end{array}\right), \\
& \sum_{k=0}^{n}\left(\begin{array}{l}
n \\
k
\end{array}\right)\left(\begin{array}{l}
m \\
k
\end{array}\right) k H_{k}=m\left(\begin{array}{c}
n+m-1 \\
n+1
\end{array}\right)\left(H_{n}-H_{n+m-1}+H_{m-2}\right), \\
& \sum_{k=0}^{n}\left(\begin{array}{l}
n \\
k
\end{array}\right)\left(\begin{array}{l}
m \\
k
\end{array}\right) k\left(H_{k}^{2}+H_{k}^{(2)}\right)=m\left(\begin{array}{c}
n+m-1 \\
n+1
\end{array}\right)\left(\left(H_{n}-H_{n+m-1}+H_{m-2}\right)^{2}+H_{n}^{(2)}-H_{n+m-1}^{(2)}+H_{m-2}^{(2)}\right), \\
& \sum_{k=0}^{n}\left(\begin{array}{l}
n \\
k
\end{array}\right)\left(\begin{array}{l}
m \\
k
\end{array}\right) k\left(H_{k}^{3}+3 H_{k} H_{k}^{(2)}+2 H_{k}^{(2)}\right) \\
& =m\left(\begin{array}{c}
n+m-1 \\
n+1
\end{array}\right)\left(\left(H_{n}-H_{n+m-1}+H_{m-2}\right)^{3}+2\left(H_{n}^{(2)}-H_{n+m-1}^{(2)}+H_{m-2}^{(2)}\right)\right. \\
& \left.\quad+3 H_{n+m-1}\left(H_{n+m-1}^{(2)}-H_{n}^{(2)}\right)+3\left(H_{n}^{(2)}-H_{n+m-1}^{(2)}+H_{m-2}^{(2)}\right)\left(H_{n}+H_{m-2}\right)\right) .
\end{aligned}
$$

Setting $r=0,1,2,3$ in Corollary 5 gives the next four identities, respectively.

Corollary 7. Let $n \geq 0$, then 


$$
\begin{aligned}
& \sum_{k=0}^{n}\left(\begin{array}{l}
n \\
k
\end{array}\right)^{2} k=n\left(\begin{array}{c}
2 n-1 \\
n+1
\end{array}\right), \\
& \sum_{k=0}^{n}\left(\begin{array}{l}
n \\
k
\end{array}\right)^{2} k H_{k}=n\left(\begin{array}{c}
2 n-1 \\
n+1
\end{array}\right)\left(H_{n}-H_{2 n-1}+H_{n-2}\right), \\
& \sum_{k=0}^{n}\left(\begin{array}{l}
n \\
k
\end{array}\right)^{2} k\left(H_{k}^{2}+H_{k}^{(2)}\right)=n\left(\begin{array}{c}
2 n-1 \\
n+1
\end{array}\right)\left(\left(H_{n}-H_{2 n-1}+H_{n-2}\right)^{2}+H_{n}^{(2)}-H_{2 n-1}^{(2)}+H_{n-2}^{(2)}\right), \\
& \sum_{k=0}^{n}\left(\begin{array}{l}
n \\
k
\end{array}\right)^{2}\left(H_{k}^{3}+3 H_{k} H_{k}^{(2)}+2 H_{k}^{(2)}\right)=n\left(\begin{array}{c}
2 n-1 \\
n+1
\end{array}\right)\left(\left(H_{n}-H_{2 n-1}+H_{n-2}\right)^{3}+2\left(H_{n}^{(2)}-H_{2 n-1}^{(2)}+H_{n-2}^{(2)}\right)\right. \\
& \left.\quad+3 H_{2 n-1}\left(H_{2 n-1}^{(2)}-H_{n}^{(2)}\right)+3\left(H_{n}^{(2)}-H_{2 n-1}^{(2)}+H_{n-2}^{(2)}\right)\left(H_{n}+H_{n-2}\right)\right) .
\end{aligned}
$$

Theorem 5. Let $n, r \geq 0, m \geq k$, then

$$
\sum_{k=0}^{n}\left(\begin{array}{l}
n \\
k
\end{array}\right)\left(\begin{array}{l}
m \\
k
\end{array}\right) \frac{P(r, n, k)}{k+1}=\frac{1}{m+1}\left(\left(\begin{array}{c}
n+m+1 \\
m
\end{array}\right) P(r, n+m+1, m)-\frac{r !|s(n+1, r)|}{(n+1) !}\right)
$$

where $s(n, h)$ are the Stirling numbers of the first kind.

Proof. By (1) and (2), we have

$$
\begin{aligned}
\sum_{k=0}^{n}\left(\begin{array}{l}
n \\
k
\end{array}\right)\left(\begin{array}{c}
m \\
k
\end{array}\right) \frac{P(r, n, k)}{k+1} & =\left[t^{n}\right] \frac{(-\ln (1-t))^{r}}{(1-t)}\left[\frac{(1+y)^{m+1}-1}{y(m+1)} \mid y=\frac{t}{1-t}\right]=\left[t^{n+1}\right] \frac{1}{m+1}\left(\frac{(-\ln (1-t))^{r}}{(1-t)^{m+1}}-(-\ln (1-t))^{r}\right) \\
& =\frac{1}{m+1}\left(\left(\begin{array}{c}
n+m+1 \\
m
\end{array}\right) P(r, n+m+1, m)-\frac{r !|s(n+1, r)|}{(n+1) !}\right),
\end{aligned}
$$

which is just the desired result.

Setting $m=n$ in Theorem 5 gives the next Corollary.

Corollary 8. Let $n, r \geq 0$, then

$$
\sum_{k=0}^{n}\left(\begin{array}{l}
n \\
k
\end{array}\right)^{2} \frac{P(r, n, k)}{k+1}=\frac{1}{n+1}\left(\left(\begin{array}{c}
2 n+1 \\
n
\end{array}\right) P(r, 2 n+1, n)-\frac{r !|s(n+1, r)|}{(n+1) !}\right)
$$

Setting $r=0,1,2,3$ in Theorem 6 gives the next Corollary.

Corollary 9. Let $n \geq 0, m \geq k$, then

$$
\begin{aligned}
& \sum_{k=0}^{n}\left(\begin{array}{l}
n \\
k
\end{array}\right)\left(\begin{array}{l}
m \\
k
\end{array}\right) \frac{1}{k+1}=\frac{1}{m+1}\left(\begin{array}{c}
n+m+1 \\
m
\end{array}\right), \\
& \sum_{k=0}^{n}\left(\begin{array}{l}
n \\
k
\end{array}\right)\left(\begin{array}{l}
m \\
k
\end{array}\right) \frac{H_{k}}{k+1}=\frac{1}{m+1}\left(\begin{array}{c}
n+m+1 \\
m
\end{array}\right)\left(H_{n}-H_{n+m+1}+H_{m}\right)+\frac{1}{(m+1)(n+1)}, \\
& \sum_{k=0}^{n}\left(\begin{array}{l}
n \\
k
\end{array}\right)\left(\begin{array}{l}
m \\
k
\end{array}\right) \frac{H_{k}^{2}+H_{k}^{(2)}}{k+1}=\frac{1}{m+1}\left(\begin{array}{c}
n+m+1 \\
m
\end{array}\right)\left(\left(H_{n}-H_{n+m+1}+H_{m}\right)^{2}+H_{n}^{(2)}-H_{n+m+1}^{(2)}+H_{m}^{(2)}\right)+\frac{2 H_{n}}{(m+1)(n+1)}, \\
& \sum_{k=0}^{n}\left(\begin{array}{l}
n \\
k
\end{array}\right)\left(\begin{array}{l}
m \\
k
\end{array}\right) \frac{H_{k}^{3}+3 H_{k} H_{k}^{(2)}+2 H_{k}^{(2)}}{k+1}= \frac{1}{m+1}\left(\begin{array}{c}
n+m+1 \\
m
\end{array}\right)\left(\left(H_{n}-H_{n+m+1}+H_{m}\right)^{3}\right. \\
&\left.+\left(2+3\left(H_{n}+H_{m}\right)\right)\left(H_{n}^{(2)}-H_{n+m+1}^{(2)}+H_{m}^{(2)}\right)+3 H_{n+m+1}\left(H_{n+m+1}^{(2)}-H_{m}^{(2)}\right)\right) \\
&+\frac{3\left(H_{n}^{(2)}+H_{m}^{(2)}\right)}{(m+1)(n+1)} .
\end{aligned}
$$


We give four applications of Corollary 9:

Corollary 10. Let $n \geq 0$, then

$$
\begin{aligned}
& \sum_{k=0}^{n}\left(\begin{array}{l}
n \\
k
\end{array}\right)^{2} \frac{1}{k+1}=\frac{1}{n+1}\left(\begin{array}{c}
2 n+1 \\
n
\end{array}\right), \\
& \sum_{k=0}^{n}\left(\begin{array}{l}
n \\
k
\end{array}\right)^{2} \frac{H_{k}}{k+1}=\frac{1}{n+1}\left(\begin{array}{c}
2 n+1 \\
n
\end{array}\right)\left(2 H_{n}-H_{2 n+1}\right)+\frac{1}{(n+1)^{2}}, \\
& \sum_{k=0}^{n}\left(\begin{array}{l}
n \\
k
\end{array}\right)^{2} \frac{H_{k}^{2}+H_{k}^{(2)}}{k+1}=\frac{1}{n+1}\left(\begin{array}{c}
2 n+1 \\
n
\end{array}\right)\left(\left(2 H_{n}-H_{2 n+1}\right)^{2}+2 H_{n}^{(2)}-H_{2 n+1}^{(2)}\right)+\frac{2 H_{n}}{(n+1)^{2}}, \\
& \sum_{k=0}^{n}\left(\begin{array}{l}
n \\
k
\end{array}\right)^{2} \frac{H_{k}^{3}+3 H_{k} H_{k}^{(2)}+2 H_{k}^{(2)}}{k+1}=\frac{1}{n+1}\left(\begin{array}{c}
2 n+1 \\
n
\end{array}\right)\left(\left(2 H_{n}-H_{2 n+1}\right)^{3}+\frac{6 H_{n}^{(2)}}{(n+1)^{2}}\right. \\
& \left.+\left(2+6 H_{n}\right)\left(2 H_{n}^{(2)}-H_{2 n+1}^{(2)}\right)+3 H_{2 n+1}\left(H_{2 n+1}^{(2)}-H_{n}^{(2)}\right)\right) .
\end{aligned}
$$

\section{Identities Involving $P(r, n, k)$ and Inverse of Binomial Coefficients}

For identities involving Harmonic numbers and inverse of binomial coefficients $\sum_{n=1}^{\infty} \frac{H_{2 n}}{n\left(\begin{array}{c}n+k \\ k\end{array}\right)}$ in given in [6].

In Section, we obtain some for $P(r, n, k)$ and binomial coefficients by means of the Riordan arrays. From these identities, we deduce some identities involving binomial coefficients, Harmonic numbers and identities related to $\zeta(2), \zeta(3)$

In [7], the inverse of a binomial coefficient is related to an integral, as follows

$$
\left(\begin{array}{l}
n \\
k
\end{array}\right)^{-1}=(n+1) \int_{0}^{1} t^{k}(1-t)^{n-k} \mathrm{~d} t
$$

From the generating function of $P(r, n, k)$ and (10), we have

Theorem 6. For $r \geq 0$ be any integer, then

$$
\sum_{n=k-1}^{\infty}\left(\begin{array}{c}
n \\
k-1
\end{array}\right)\left(\begin{array}{c}
n+k \\
k
\end{array}\right)^{-1} \frac{P(r, n, k-1)}{n+k+1}=-\frac{r !}{k} \sum_{j=1}^{k}\left(\begin{array}{c}
k \\
j
\end{array}\right) \frac{(-1)^{j}}{j^{r}}
$$

Proof. From (1) and (10), we obtain

$$
\begin{aligned}
\sum_{n=k-1}^{\infty}\left(\begin{array}{c}
n \\
k-1
\end{array}\right)\left(\begin{array}{c}
n+k \\
k
\end{array}\right)^{-1} \frac{P(r, n, k-1)}{n+k+1} & =\sum_{n=k-1}^{\infty}\left(\begin{array}{c}
n \\
k-1
\end{array}\right) P(r, n, k-1) \int_{0}^{1} t^{k}(1-t)^{n} \mathrm{~d} t \\
& =\int_{0}^{1} t^{k} \frac{(1-t)^{k-1}(-\ln (1-(1-t)))^{r}}{(1-(1-t))^{k}} \mathrm{~d} t \\
& =\int_{0}^{1}(1-t)^{k-1}(-\ln t)^{r} \mathrm{~d} t \\
& =-\frac{r !}{k} \sum_{j=1}^{k}\left(\begin{array}{c}
k \\
j
\end{array}\right) \frac{(-1)^{j}}{j^{r}} .
\end{aligned}
$$

This gives (11).

Corollary 11 Setting $r=0,1,2,3$ in Theorem 6, The following relation holds:

$$
\sum_{n=k-1}^{\infty}\left(\begin{array}{c}
n \\
k-1
\end{array}\right)\left(\begin{array}{c}
n+k \\
k
\end{array}\right)^{-1} \frac{1}{n+k+1}=\frac{1}{k}
$$




$$
\begin{gathered}
\sum_{n=k-1}^{\infty}\left(\begin{array}{c}
n \\
k-1
\end{array}\right)\left(\begin{array}{c}
n+k \\
k
\end{array}\right)^{-1} \frac{H_{n}}{n+k+1}=\frac{1}{k}\left(H_{k}+H_{k-1}\right) \\
\sum_{n=k-1}^{\infty}\left(\begin{array}{c}
n \\
k-1
\end{array}\right)\left(\begin{array}{c}
n+k \\
k
\end{array}\right)^{-1} \frac{H_{n}^{2}-H_{n}^{(2)}}{n+k+1}=\frac{1}{k}\left(\left(H_{k}+H_{k-1}\right)^{2}+H_{k}^{(2)}-H_{k-1}^{(2)}\right) \\
\sum_{n=k-1}^{\infty}\left(\begin{array}{c}
n \\
k-1
\end{array}\right)\left(\begin{array}{c}
n+k \\
k
\end{array}\right)^{-1} \frac{H_{n}^{3}+2 H_{n}^{(2)}-3 H_{n} H_{n}^{(2)}}{n+k+1} \\
=\frac{1}{k}\left(\left(H_{k}+H_{k-1}\right)^{3}+2\left(H_{k-1}^{(2)}+H_{k}^{(3)}\right)+3\left(H_{k}+H_{k-1}\right)\left(H_{k}^{(2)}-H_{k-1}^{(2)}\right)\right)
\end{gathered}
$$

Setting $k=1,2$ in Corollary 11, gives the next identities.

Corollary 12 The following relation holds

$$
\begin{gathered}
\sum_{n=0}^{\infty} \frac{1}{(n+1)(n+2)}=1 \\
\sum_{n=0}^{\infty} \frac{H_{n}}{(n+1)(n+2)}=1 \\
\sum_{n=0}^{\infty} \frac{H_{n}^{2}-H_{n}^{(2)}}{(n+1)(n+2)}=2 \\
\sum_{n=0}^{\infty} \frac{H_{n}^{3}+2 H_{n}^{(2)}-3 H_{n} H_{n}^{(2)}}{(n+1)(n+2)}=6 \\
\sum_{n=1}^{\infty} \frac{n}{(n+1)(n+2)(n+3)}=\frac{1}{4} \\
\sum_{n=1}^{\infty} \frac{n H_{n}}{(n+1)(n+2)(n+3)}=\frac{5}{8} \\
\sum_{n=1}^{\infty} \frac{n\left(H_{n}^{2}-H_{n}^{(2)}\right)}{(n+1)(n+2)(n+3)}=\frac{13}{8} \\
\sum_{n=1}^{\infty} \frac{n\left(H_{n}^{3}+2 H_{n}^{(2)}-3 H_{n} H_{n}^{(2)}\right)}{(n+1)(n+2)(n+3)}=\frac{87}{16}
\end{gathered}
$$

Corollary 13. The following relation holds

$$
\begin{gathered}
\sum_{n=1}^{\infty} \frac{1}{(n+1)(n+2)(n+3)}=\frac{1}{4} \\
\sum_{n=1}^{\infty} \frac{H_{n}}{(n+1)(n+2)(n+3)}=\frac{1}{8} \\
\sum_{n=1}^{\infty} \frac{H_{n}^{2}-H_{n}^{(2)}}{(n+1)(n+2)(n+3)}=\frac{1}{8} \\
\sum_{n=1}^{\infty} \frac{H_{n}^{3}+2 H_{n}^{(2)}-3 H_{n} H_{n}^{(2)}}{(n+1)(n+2)(n+3)}=\frac{3}{16}
\end{gathered}
$$

Proof. (16) minus(20) give (24); (17) minus (21), (18) minus (22) and (19) minus (23), yields (25), (26) and (27), respectively. 
Leonhard Euler (1707-1783) had already stated the equation

$$
\zeta(s)=\sum_{n=1}^{\infty} \frac{1}{n^{s}}=\prod_{p \text { prime }} \frac{1}{1-p^{-s}}
$$

Recall the Euler sum identities [8] [9].

$$
\sum_{n=k}^{\infty} \frac{H_{n}}{n^{2}}=2 \zeta(3), \quad \sum_{n=k}^{\infty} \frac{H_{n}}{n^{3}}=\frac{5}{4} \zeta(4), \quad \sum_{n=k}^{\infty} \frac{H_{n}}{n(n+1)}=\zeta(2), \quad \sum_{n=k}^{\infty} \frac{H_{n}}{(n+1)^{2}}=\zeta(3)
$$

The next, we gives identities related to $\zeta(2), \quad \zeta(3)$

For completeness we supply proofs:

$$
\begin{aligned}
\sum_{n=1}^{\infty} \frac{H_{n}^{2}}{(n+1)(n+2)}= & \sum_{n=1}^{\infty}\left(\frac{1}{n+1}\left(H_{n-1}+\frac{1}{n}\right)^{2}-\frac{H_{n}^{2}}{n+2}\right)=\sum_{n=1}^{\infty}\left(\frac{H_{n-1}^{2}}{n+1}-\frac{H_{n}^{2}}{n+2}+\frac{2 H_{n-1}}{n(n+1)}+\frac{1}{n^{2}(n+1)}\right) \\
= & 2 \sum_{n=1}^{\infty} \frac{H_{n}}{(n+1)(n+2)}+\sum_{n=1}^{\infty} \frac{1}{n^{2}}-\sum_{n=1}^{\infty} \frac{1}{n(n+1)}=1+\zeta(2) . \\
\sum_{n=1}^{\infty} \frac{H_{n}}{(n+1)^{2}(n+3)} & =\frac{1}{2} \sum_{n=1}^{\infty} \frac{H_{n}}{(n+1)^{2}}+\frac{1}{4} \sum_{n=1}^{\infty}\left(\frac{H_{n}}{(n+3)}-\frac{H_{n}}{(n+1)}\right) \\
& =\frac{1}{2} \sum_{n=1}^{\infty} \frac{H_{n}}{(n+1)^{2}}+\frac{1}{4}\left(\sum_{n=1}^{\infty} \frac{H_{n}}{(n+3)}-\sum_{n=1}^{\infty} \frac{1}{(n+1)}\left(H_{n-2}+\frac{1}{(n-1)}+\frac{1}{n}\right)\right) \\
& =\frac{1}{2} \sum_{n=1}^{\infty} \frac{H_{n}}{(n+1)^{2}}-\frac{1}{4}\left(\sum_{n=1}^{\infty} \frac{1}{(n+1) n}-\sum_{n=2}^{\infty} \frac{1}{(n+1)(n-1)}\right)=\frac{1}{2} \zeta(2)-\frac{7}{16} .
\end{aligned}
$$

Similarly, we obtain summation formulas related $\zeta(2), \quad \zeta(3)$

$$
\begin{gathered}
\sum_{n=1}^{\infty} \frac{H_{n}^{2}}{(n+1)(n+3)}=\frac{1}{2}(1+\zeta(2)) \\
\sum_{n=1}^{\infty} \frac{H_{n}^{3}}{(n+1)(n+2)}=1+2 \zeta(2)+4 \zeta(3)
\end{gathered}
$$

By (18) and (28), (19) and (31), we have

$$
\begin{gathered}
\sum_{n=1}^{\infty} \frac{H_{n}^{(2)}}{(n+1)(n+2)}=\zeta(2)-1 \\
\sum_{n=1}^{\infty} \frac{H_{n} H_{n}^{(2)}}{(n+1)(n+2)}=\frac{4}{3}(\zeta(2)+\zeta(3))-1
\end{gathered}
$$

Similarly, for completeness we supply a proof:

$$
\begin{aligned}
\sum_{n=1}^{\infty} \frac{H_{n}^{3}}{(n+1)(n+2)(n+3)} & =\frac{1}{2} \sum_{n=1}^{\infty} \frac{H_{n}^{3}}{(n+1)(n+2)}-\frac{1}{2} \sum_{n=1}^{\infty} \frac{H_{n}^{3}}{(n+2)(n+3)} \\
& =\frac{1}{2} \sum_{n=1}^{\infty} \frac{H_{n}^{3}}{(n+1)(n+2)}-\frac{1}{2} \sum_{n=1}^{\infty}\left(\frac{1}{n+2}\left(H_{n-1}+\frac{1}{n}\right)^{3}-\frac{H_{n}^{3}}{n+3}\right) \\
& =\frac{1}{2}(1+2 \zeta(2)+4 \zeta(3))-\frac{3}{2} \sum_{n=1}^{\infty} \frac{H_{n}^{2}}{(n+1)(n+3)}-\frac{3}{2} \sum_{n=1}^{\infty} \frac{H_{n}}{(n+1)^{2}(n+3)} \\
& =\frac{1}{4}\left(\zeta(2)+5 \zeta(3)+\frac{13}{8}\right) .
\end{aligned}
$$


By (28) minus (30), we get

$$
\sum_{n=1}^{\infty} \frac{H_{n}^{2}}{(n+1)(n+2)(n+3)}=\sum_{n=1}^{\infty} \frac{H_{n}^{2}}{(n+1)(n+2)}-\sum_{n=1}^{\infty} \frac{H_{n}^{2}}{(n+2)(n+3)}=\frac{1}{2}(1+\zeta(2))
$$

Applying (25) and (34), (26) and (32), we have

$$
\sum_{n=1}^{\infty} \frac{H_{n}^{(2)}}{(n+1)(n+2)(n+3)}=\frac{1}{2} \zeta(2)+\frac{3}{8}, \quad \sum_{n=1}^{\infty} \frac{H_{n} H_{n}^{(2)}}{(n+1)(n+2)(n+3)}=\frac{5}{12}(\zeta(2)+\zeta(3))+\frac{31}{96}
$$

\section{Asymptotics}

Theorem 7 For $r \geq 1$ be any integer, as $n \rightarrow \infty$, we have

$$
\sum_{k=0}^{n}\left(\begin{array}{l}
n \\
k
\end{array}\right)\left(\begin{array}{l}
m \\
k
\end{array}\right) P(r, n, k) \sim \begin{cases}\frac{n^{m-2} \ln ^{r} n}{\Gamma(m-1)}, & m \notin\{1,0,-1-2 \cdots\} ; \\
\frac{(-1)^{-m+1} r(-m+1) ! \ln ^{r-1} n}{n^{2-m}}, & m \in\{1,0,-1-2 \cdots\} .\end{cases}
$$

Proof. By Lemma 1, we have

$$
\sum_{k=0}^{n}\left(\begin{array}{l}
n \\
k
\end{array}\right)\left(\begin{array}{l}
m \\
k
\end{array}\right) P(r, n, k)=\left[t^{n}\right] \frac{(-\ln (1-t))^{r}}{(1-t)^{m+1}} \sim \begin{cases}\frac{n^{m-2} \ln ^{r} n}{\Gamma(m-1)}, & m \notin\{1,0,-1-2 \cdots\} ; \\
\frac{(-1)^{-m+1} r(-m+1) ! \ln ^{r-1} n}{n^{2-m}}, & m \in\{1,0,-1-2 \cdots\} .\end{cases}
$$

and this complete the proof.

Similarly, we can obtain the next Theorem.

Theorem 8. Let $r \geq 1$ be any integer, as $n \rightarrow \infty$, we have

$$
\sum_{k=0}^{n}\left(\begin{array}{l}
n \\
k
\end{array}\right)\left(\begin{array}{l}
m \\
k
\end{array}\right) k P(r, n, k) \sim \begin{cases}\frac{m n^{m-2} \ln ^{r} n}{\Gamma(m-1)}, & m \notin\{1,0,-1-2 \cdots\} ; \\
\frac{m(-1)^{-m+1} r(-m+1) ! \ln ^{r-1} n}{n^{2-m}}, & m \in\{1,0,-1-2 \cdots\} .\end{cases}
$$

Theorem 9. For $r \geq 1$ be any integer, as $n \rightarrow \infty$, we have

$$
\sum_{k=0}^{n}\left(\begin{array}{l}
n \\
k
\end{array}\right)\left(\begin{array}{l}
m \\
k
\end{array}\right) \frac{P(r, n, k)}{k+1} \sim \begin{cases}\frac{1}{m+1}\left(\frac{n^{m-2} \ln ^{r} n}{\Gamma(m-1)}-\frac{r \ln ^{r-1} n}{n+1}\right), & m \notin\{1,0,-1-2 \cdots\} ; \\
\frac{1}{m+1}\left(\frac{(-1)^{-m+1} r(-m+1) ! \ln ^{r-1} n}{n^{2-m}}-\frac{r n^{r-1} n}{n+1}\right), & m \in\{1,0,-1-2 \cdots\} .\end{cases}
$$

Proof. By Lemma 1, we have

$$
\begin{aligned}
& \sum_{k=0}^{n}\left(\begin{array}{l}
n \\
k
\end{array}\right)\left(\begin{array}{l}
m \\
k
\end{array}\right) \frac{P(r, n, k)}{k+1}=\left[t^{n+1}\right] \frac{1}{m+1}\left(\frac{(-\ln (1-t))^{r}}{(1-t)^{m+1}}-(-\ln (1-t))^{r}\right) \\
& \sim \begin{cases}\frac{1}{m+1}\left(\frac{n^{m-2} \ln ^{r} n}{\Gamma(m-1)}-\frac{r \ln ^{r-1} n}{n+1}\right), & m \notin\{1,0,-1-2 \cdots\} ; \\
\frac{1}{m+1}\left(\frac{(-1)^{-m+1} r(-m+1) ! \ln ^{r-1} n}{n^{2-m}}-\frac{r \ln ^{r-1} n}{n+1}\right), & m \in\{1,0,-1-2 \cdots\} .\end{cases}
\end{aligned}
$$


this give (38).

Theorem 10. For $r \geq 0$ be any integer, as $k \rightarrow \infty$, we have

$$
\sum_{n=k-1}^{\infty}\left(\begin{array}{c}
n \\
k-1
\end{array}\right)\left(\begin{array}{c}
n+k \\
k
\end{array}\right)^{-1} \frac{P(r, n, k-1)}{n+k+1} \sim \frac{1}{k} \sum_{j=0}^{r}\left(\begin{array}{l}
r \\
j
\end{array}\right)(-1)^{j} \Gamma^{(j)}(1) \ln ^{r-j} k+O\left(\frac{r ! \ln k}{k^{2}}\right)
$$

Proof. By Corollary 3 of [10], immediately complete the proof of Theorem 10.

\section{Acknowledgements}

The author would like to thank an anonymous referee whose helpful suggestions and comments have led to much improvement of the paper. The research is supported by the Natural Science Foundation of China under Grant 11461050 and Natural Science Foundation of Inner Mongolia under Grant 2012MS0118.

\section{References}

[1] Zave, D.A. (1976) A Series Expansion Involving the Harmonic Numbers. Information Processing Letters, 5, $75-77$. http://dx.doi.org/10.1016/0020-0190(76)90068-5

[2] Spiess, J. (1990) Some Identities Involving Harmonic Numbers. Mathematics Computation, 55, 839-863. http://dx.doi.org/10.1090/S0025-5718-1990-1023769-6

[3] Brietzke, E.H.M. (2008) An Identity of Andrews and a New Method for the Riordan Array Proof of Combinatorial Identities. Discrete Mathematics, 308, 4246-4262. http://dx.doi.org/10.1016/j.disc.2007.08.050

[4] Wang, W. and Wang, T (2008) Generalized Riordan Arrays. Discrete Mathematics, 308, 6466-6500. http://dx.doi.org/10.1016/j.disc.2007.12.037

[5] Flajolet, P., Fusy, E., Gourdon, X., Panario, D. and Pouyanne, N. (2006) A Hybrid of Darboux’s Method and Singularity Analysis in Combinatorial Asymptotics. The Electronic Journal of Combinatorics, 13.

[6] Sofo, A. (2012) Euler Related Sums. Mathematical Sciences, 6, 10.

[7] Sury, B. (1993) Sum of the Reciprocals of the Binomial Coefficients. European Journal of Combinatorics, 14, 351353. http://dx.doi.org/10.1006/eujc.1993.1038

[8] Jonathan, M. (2009) Borwein and O-Yeat Chang. Duallity in Tails of Multiple-Zeta Values, 54, 2220-2234.

[9] David, B. and Borwein, J.M. (1995) On an Intrguing Integral and Some Series Relate to $\zeta$ (4). Proceedings of the American Mathematical Society, 123, 1191-1198.

[10] Flajolet, P. and Sedgewick, R. (1995) Mellin Transforms Asymptotics: Finite Differences and Rice’s Integrals. Theoretical Computer Science, 144, 101-124. http://dx.doi.org/10.1016/0304-3975(94)00281-M 